\title{
Organização de serviços de saúde na eliminação da Hanseníase em municípios do Estado de São Paulo
}

\author{
Health services organization on the elimination of Leprosy in municipalities of São Paulo State \\ La organización de los servicios de salud en la eliminación de la Lepra en municipios del Estado de São Paulo
}

\author{
Lúcia Maria Frazão Helene', Elisete Silva Pedrazzani", Cleide Lavieri Martins"II, \\ Carmen Silva de Campos Almeida Vieiralv, Adriana Jimenez Pereiral \\ 'Universidade de São Paulo. Escola de Enfermagem, Departamento de Saúde Coletiva. São Paulo, SP \\ I'Universidade Federal de São Carlos. Departamento de Enfermagem. São Carlos, SP \\ "'Iniversidade de São Paulo. Faculdade de Saúde Pública, Departamento de Prática de Saúde Pública. São Paulo, SP \\ ${ }^{N}$ Universidade de Taubaté. Departamento de Enfermagem. Taubaté,SP
}

Submissão: $30 / 08 / 2008$

Aprovação: 20/10/2008

\section{RESUMO}

Este trabalho tem como objetivo analisar as ações de controle da hanseníase desenvolvidas em nove municípios do Estado de São Paulo e uma coordenadoria de saúde da Capital, selecionados a partir de sua população, localização geográfica e coeficientes de prevalência. Foi aprovado pelo Comitê de Ética no $514 / 05$ e foram aplicados instrumentos por meio de entrevistas em 59 profissionais e 17 interlocutores. Os dados foram analisados utilizando o programa SPSS (Statistical Package for Social Scientists). Verificou-se Que a prevalência apresenta declínio e a detecção permanece estável. As ações de prevenção de incapacidade física, educação em saúde e a busca ativa são pouco desenvolvidas. Os profissionais mais capacitados encontram-se nas unidades de referência, há fragilidade Quanto ao trabalho em eQuipe. Descritores: Hanseníase; Controle de Doenças Transmissíveis, Organização de Serviços de Saúde

\begin{abstract}
The present study aims to analyze actions to control Hansen's disease developed in nine municipalities of the State of São Paulo and one of the health authorities of the city of São Paulo, selected based on their population, geographic location and prevalence coefficients. The study was approved by the Ethics Committee no $514 / 05$ and instruments were administered by means of interviews to 59 professionals, 17 interlocutors and 9 managers. Data were analyzed using the program SPSS (Statistical Package for Social Scientists). It was verified that prevalence is on the decline and detection remains stable. The physical incapacity prevention actions, health education and active search have not been satisfactorily developed. The most Qualified professionals are in the reference units and teamwork is fragile.
\end{abstract}

Descriptors: Leprosy; Control of transmissible diseases; Health Services Organization.

\section{RESUMEN}

El objetivo de este trabajo fue analizar las acciones de control de la Lepra realizadas en nueve Municipios del Estado de Sao Paulo y en una coordinación de salud de la capital, seleccionados a partir de su población, localización geográfica y coeficientes de prevalencia. Este estudio recibió aprobación del Comité de Ética n ${ }^{\circ}$ 1 14/05. Se entrevistaron 59 profesionales y 17 coordinadores de área. Para el análisis de los datos se utilizó el programa SPSS (Statistical Package for Social Scientists). Se verificó un declive en la prevalencia, pero la detección permaneció estable; las acciones de prevención de incapacidad física, educación en salud y la busca activa fueron poco ejecutadas; los profesionales más capacitados pertenecían a las unidades de referencia. Hubo fragilidad en cuanto al trabajo en equipo.

Descriptores: Lepra; Control de enfermedades transmisibles; Organización de servicios de salud.

Pesquisa financiada pelo Conselho Nacional de Desenvolvimento Científico e Tecnológico (CNPQ) - Processo 400830/2005-I. Edital MCT-CNPQ/MSSCTIE - no 35/2005 


\section{INTRODUÇÃO}

Atualmente, com os encaminhamentos coordenados pelo Ministério da Saúde do Brasil (MS) e Organização Mundial da Saúde (OMS) visando a eliminação da hanseníase em nosso país, muitos esforços estão sendo empreendidos para Que possamos atingir essa meta. O Brasil tem como meta acordada com a OMS a eliminação da hanseníase como problema de saúde pública até o final do ano de 2010 , onde se espera Que todos os municípios atinjam uma taxa de prevalência de menos de 1 doente por 10.000 habitantes $^{(1)}$. Para alcançar esta meta há também necessidade urgente do aumento de cobertura das ações de controle da doença para todas as unidades de saúde dos municípios endêmicos, como parte de uma política geral de descentralização Que vem sendo desenvolvida pelo Ministério da Saúde, tendo como estratégia o Programa de Saúde da Família (PSF) ${ }^{(2)}$.

Para tanto, a Secretaria de Estado da Saúde de São Paulo preconizou a implementação da Resolução SS - 130, publicada no Diário Oficial do Estado em 10 de outubro de 2001, páginas 19 à 22, Que aprova a Norma Técnica Que estabelece as Diretrizes e Estratégias para as Ações de Controle da Hanseníase e Preconiza Esquemas de Tratamento ${ }^{(3)}$, e Que contém várias diretrizes para as ações de controle da hanseníase.

Essa norma incide em aspectos relevantes, dentre os Quais: o acesso à assistência integral às pessoas com suspeita e diagnosticadas com hanseníase, de acordo com as suas necessidades; assistência essa Que deve ser desenvolvida por trabalhadores com preparo específico sobre hanseníase e atualização permanente; condições materiais de trabalho, o espaço físico/número de doentes nos serviços de saúde e os equipamentos e os materiais físicos e pedagógicos nele utilizados devem facilitar a assistência realizada.

Mais recentemente foi publicado o Plano de Combate à Hanseníase no Estado de São Paulo - 2006 a $2010^{(4)}$, Que contém as seguintes metas e diretrizes: aprimorar o sistema de avaliação da endemia por município; implementar as ações de detecção e tratamento nos municípios endêmicos; implementar as ações de vigilância nos municípios com prevalência de eliminação; reorganizar o sistema de referência e contra-referência, local e regionalizado; prevenir e tratar incapacidades físicas detectadas nos doentes; implementar as ações sociais e educativas e reorganizar o sistema de informações.

Assim alguns desafios nos apresentam, especialmente, com a Questão da descentralização e municipalização da saúde Que conferiu ao município a competência de normalizar os serviços de saúde.

Com a descentralização dos serviços para os Municípios; e a regionalização e hierarQuização da rede de serviços de saúde o sistema de saúde passa por uma reformulação, surge um novo modelo de gestão social participativa. Com a regionalização, haveria uma distribuição espacial de serviços de saúde dos diversos níveis de complexidade organizados para atender a população de uma determinada região, pautado em uma forma de organização Que contemple suporte técnico e de recursos humanos, permitindo suficiência de recursos e poder decisório definido ${ }^{(5)}$.

O Estado de São Paulo teve uma situação peculiar no Que se refere a uma importante redução do número de doentes em tratamento, caracterizando-se atualmente, como um Estado em que muitos municípios apresentam coeficientes de prevalência de eliminação (abaixo de I doente/10.000 habitantes). Ao mesmo tempo apresenta municípios em Que a hanseníase ainda persiste com altos coeficientes de prevalência e detecção, são regiões Que fazem divisa com os estados de Mato Grosso do Sul e Minas Gerais, municípios considerados em alerta com prevalência zero e onde a hanseníase foi considerada eliminada, e aQueles Que mantêm uma média prevalência da doença. Esta situação especial nos remete a necessidade de discussões e reflexões sobre a descentralização e municipalização da saúde considerando-se as diferentes realidades municipais num contexto polêmico como o encontrado em nosso Estado.

Dessa forma, neste estudo, pretendeu-se identificar e discutir estratégias Que possam subsidiar os serviços de saúde no seu trabalho junto à população e aos doentes com hanseníase com vistas à eliminação da doença no Estado de São Paulo.

\section{OBJETIVO}

Analisar comparativamente as ações de controle e eliminação da hanseníase desenvolvidas em municípios do Estado de São Paulo, no período de 2001 a 2005, com ênfase na organização dos serviços e na construção de uma nova assistência à hanseníase de Qualidade com vistas à eliminação da doença, incluindo a reabilitação e reinserção dos doentes na sociedade.

\section{METODOLOGIA}

O presente trabalho é um estudo longitudinal retrospectivo e foi desenvolvido em municípios do Estado de São Paulo, selecionados por meio de um diagnóstico preliminar da situação epidemiológica da hanseníase a partir dos coeficientes de prevalência, do tamanho populacional e da localização geográfica dos municipios em relação ao Estado. Dessa forma, esse diagnóstico preliminar possibilitou além da seleção dos municípios, a dos serviços de saúde desses municipios e dos sujeitos de pesquisa.

Para a seleção dos municípios observou-se os seguintes critérios:

a) Características relacionadas ao grau de prioridade dos municípios para o controle da Hanseníase (O Ministério da Saúde estabelece que os municípios podem ser classificados em: municípios Prioritários PI: - mais de 20.000 habitantes e coeficiente de detecção (2003) maior Que 3,0/10.000 hab.; e em municípios Prioritários - P2: - mais de 20.000 habitantes e coeficiente de detecção (2003) maior ou igual a I,0/10.000 hab. e menor do Que 3,0/10.000 habitantes): foram escolhidos três municípios do interior de cada nível de prioridade e a capital que é considerada Prioridade 1;

b) Características relacionadas à situação epidemiológica: foram contemplados 3 municípios com coeficientes de prevalência alta, 3 com média e 3 com baixa, e o município de São Paulo.

c) Características relacionadas ao tamanho da população: foram selecionados, 3 municípios de pequeno porte populacional (20 a 30.000 habitantes); 3 de médio ( 150 a 200.000 habitantes); e 3 de grande (acima de 200.000 habitantes); além da cidade de São Paulo. Optamos não investigar municípios com menos de 20.000 habitantes pela probalilidade destes não possuirem serviços organizados para o atendimento.

d) Características relacionadas à localização geográfica no Estado: foram selecionados três municípios de cada uma das regiões - Sul, Norte e Oeste - contemplando diferentes áreas geográficas.

A conjugação dos critérios acima levou a escolha de 10 municípios: 
na região Sul do estado de São Paulo: Parieuera-açu, Sorocaba e Pilar do Sul; na região Norte do estado de São Paulo: São José do Rio Preto, Catanduva e José Bonifácio; na região Oeste do estado de São Paulo: Dracena, Presidente Prudente e Bauru; e o Município de São Paulo.

Para a definição dos serviços de saúde pesquisados, foram considerados: a Quantidade de unidades básicas de saúde (UBS) no município e o tipo de atividades executadas pelas UBS em relação à hanseníase. Foram incluídas no estudo as UBS de maior complexidade em atendimento, chamadas de referência, além das UBS de menor complexidade, escolhidas por meio de sorteio objetivando uma amostra de 10\% dos serviços existentes em cada município. Dessa forma foram selecionados 7 serviços de saúde na região Sul do estado de São Paulo; 7 na região Norte; 8 na região Oeste; e 6 no município de São Paulo. Pelas suas características e dimensões populacionais, o estudo na cidade de São Paulo foi realizado em uma das cinco Coordenadorias Regionais de Saúde existentes, a Coordenadoria Regional de Saúde Leste. Desta Coordenadoria, formada por 7 Supervisões de Saúde foram selecionadas a Supervisão Itaquera - Que possui a UBS mais antiga na região no Que diz repeito ao desenvolvimento das atividades de assistência e controle da Hanseníase -, e a de Ermelino Matarazzo que tem o maior coeficiente de detecção.

\section{Coleta de dados}

A partir da seleção dos municípios e serviços, o trabalho de campo teve início com os contatos com os coordenadores e responsáveis pelos serviços de saúde. Em seguida, as etapas de visitas às UBS onde foi possível identificar os documentos pertinentes, realizar as entrevistas e o levantamento dos dados epidemiológicos e operacionais do Programa de Controle da Hanseníase (PCH). Os interlocutores, os gestores municipais e os profissionais de saúde dos municipios e serviços selecionados concordaram em participar do estudo. Este artigo restringe-se à análise das entrevistas com os interlocutores e profissionais de saúde. A análise das entrevistas com os gestores será objeto de outro artigo. Os dados epidemiológicos, de 2001 a 2005, foram obtidos com base nos boletins epidemiológicos produzidos pelo Programa Estadual de Controle da Hanseníase e pela Secretaria Municipal de Saúde.

Foram realizadas 76 entrevistas com 59 profissionais assistenciais de diferentes formações e 17 interlocutores Que são responsáveis por fazer a interlocução entre profissionais Que prestam assistência nos serviços de saúde e a área de gestão do $\mathrm{PCH}$, atuando como facilitador/articulador.

\section{Análise dos dados}

Os dados Quantitativos foram armazenados em banco de dados e processados pelo programa SPSS (Statistical Package for Social Scientists), com produção de gráficos e tabelas. As informações obtidas por meio das Questões abertas das entrevistas foram agrupadas conforme semelhança de conteúdo das respostas.

\section{Aspectos éticos}

Foram seguidas as diretrizes e normas do Conselho Nacional de Saúde, Resolução no ${ }^{\circ}$ 196. O projeto de pesQuisa foi aprovado pelo Comitê de Ética da Escola de Enfermagem da Universidade de São
Paulo, processo no5 14/2005/CEP-EEUSP.

Por meio do Termo de Consentimento Livre e Esclarecido, foi solicitada a assinatura dos sujeitos de pesquisa e fornecida explicação para o consentindo em participar livre e voluntariamente do estudo, em ter a entrevista gravada mantendo-se o anonimato do entrevistado.

\section{RESULTADOS E DISCUSSÃO}

\section{Situação Epidemiológica da Hanseníase nos Municípios Estudados}

De acordo com o Programa Estadual de Controle da Hanseníase o Estado de São Paulo apresenta diferentes situações epidemiológicas e operacionais em seus municípios e, dessa forma, são necessários diferentes níveis de complexidade das ações para o controle da hanseníase ${ }^{(6)}$.

Neste estudo, os indicadores foram calculados com base nas informações existentes na Divisão Técnica de Hanseníase do Centro de Vigilância Epidemiológica Prof. "Alexandre Vranjac" (CVE) da Secretaria de Estado da Saúde de São Paulo que dispõe de banco de dados contendo as informações de hanseníase de todo o Estado, por Diretoria Regional de Saúde (DIR) e por município.

No Estado de São Paulo o índice de prevalência da hanseníase em 2002 foi de 1,4l/10.000 habitantes, com 5.378 doentes em registro ativo; o número de casos detectados nesse mesmo ano foi 2.765 com um coeficiente de detecção de 0,72. Em 2005 os valores encontrados no Estado correspondem a 0,44/10.000 hab. com o alcance da meta de eliminação.

Conforme dados apresentados na Tabela I, no período compreendido entre 2001 e 2005, a maioria dos municípios estudados apresentou uma diminuição significativa dos coeficientes de prevalência dos casos de hanseníase, e essa diminuição foi mais acentuada no ano de 2004.

O município de José Bonifácio apresentou os maiores índices de prevalência durante todo o período analisado, entretanto, vem passando por importantes alterações com diminuição acentuada nos coeficientes, assim, tinha 9,91 de prevalência no ano de 2003 passando para 2,82 no ano de 2005. Da mesma forma, em 2002, o município de Dracena tinha 9, 10 de prevalência, passando para 6,87\% em 2003 e alcançou o coeficiente de 4,88 no ano de 2005.

Ainda pode-se verificar na Tabela 1, Que a prevalência dos municípios de Bauru e Pilar do Sul representaram o menor incremento de participação na prevalência dos municípios estudados no período. Quanto ao coeficiente de casos novos segundo faixa etária, foi verificada a existência de menores de idade com hanseníase em todos os municípios estudados, entretanto com reduzida percentagem, sendo Que, um município (Sorocaba) apresentou um número maior de menores de 15 anos de idade com hanseníase.

Observou-se que dentre os casos novos detectados, segundo o grupo bacilar, prevaleceu os casos multibacilares (MB), nos municípios de São losé do Rio Preto, Bauru e São Paulo, o Que leva a pensar na possibilidade de que o diagnóstico ainda é realizado tardiamente.

Quanto à forma clínica, houve predomínio da Tuberculóide na maioria dos municípios, o Que indica uma evolução tardia da doença. Destaca-se que foi verificado um reduzido número de casos novos diagnosticados na forma clínica Indeterminada, na maioria dos municípios. 
Tabela 1. Prevalência de hanseníase, 2001 a 2005, de acordo com os municípios estudados. São Paulo/SP, 2007.

\begin{tabular}{lccccc}
\hline \multirow{2}{*}{ Municípios } & \multicolumn{5}{c}{ Coeficiente de Prevalência por $\mathbf{1 0 . 0 0 0}$ habitantes } \\
\cline { 2 - 6 } & 2001 & $\mathbf{2 0 0 2}$ & $\mathbf{2 0 0 3}$ & $\mathbf{2 0 0 4}$ & $\mathbf{2 0 0 5}$ \\
\hline Bauru & 0,56 & 0,58 & 1,02 & 0,31 & 0,57 \\
Presidente Prudente & 3,96 & 3,40 & 3,46 & 1,51 & 2,99 \\
Dracena & 9,37 & 9,10 & 6,87 & 6,37 & 4,88 \\
São José do Rio Preto & 1,03 & 1,57 & 1,49 & 0,28 & 1,06 \\
José Bonifácio & 4,11 & 7,39 & 9,91 & 3,58 & 2,82 \\
Catanduva & 3,07 & 1,83 & 1,36 & 1,25 & 1,56 \\
Pariquea-Açu & 2,20 & 2,69 & 4,73 & 2,57 & 0,98 \\
Pilar do Sul & 1,63 & 0,40 & 0,39 & 0,00 & 0,37 \\
Sorocaba & 1,93 & 1,58 & 1,29 & 0,81 & 0,88 \\
São Paulo (coord leste) & $*$ & 0,67 & 0,73 & 0,68 & 0,69 \\
\hline
\end{tabular}

Fonte: Divisão Técnica de Vigilância Epidemiológica da Hanseníase (DVE/CCD/SES) * Dado não obtido nas fontes consultadas.

Tabela 2. Proporção de casos com Incapacidade Física, Grau I + II, entre os casos novos detectados, 2001 a 2005, segundo os municípios estudados. São Paulo/SP. 2007.

\begin{tabular}{|c|c|c|c|c|c|c|c|c|c|c|}
\hline \multirow{3}{*}{ Municípios } & \multicolumn{10}{|c|}{ Proporção de casos novos com incapacidade física grau I e II } \\
\hline & \multicolumn{2}{|c|}{2001} & \multicolumn{2}{|c|}{2002} & \multicolumn{2}{|c|}{2003} & \multicolumn{2}{|c|}{2004} & \multicolumn{2}{|c|}{2005} \\
\hline & $\mathrm{n}$ & $\%$ & $\mathrm{~N}$ & $\%$ & $\mathrm{n}$ & $\%$ & $\mathrm{n}$ & $\%$ & $\mathrm{n}$ & $\%$ \\
\hline Bauru & 4 & 44,4 & 6 & 54,5 & 12 & 57,1 & 5 & 45,4 & 7 & 46,6 \\
\hline Presidente Prudente & 28 & 50,0 & 22 & 44,0 & 23 & 50,0 & 20 & 51,3 & 11 & 34,4 \\
\hline Dracena & 16 & 50,0 & 10 & 41,7 & 10 & 41,9 & 8 & 28,6 & 4 & 20,0 \\
\hline São José do Rio Preto & 14 & 28,6 & 11 & 28,2 & 3 & 12,5 & 11 & 28,9 & 7 & 33,3 \\
\hline José Bonifácio & 6 & 75,0 & 9 & 60,0 & 7 & 41,2 & 4 & 40,0 & 2 & 28,6 \\
\hline Catanduva & 1 & 6,7 & 1 & 11,1 & - & 0,0 & - & 0,0 & 1 & 7,1 \\
\hline PariQuea-Açu & - & 0,0 & - & 0,0 & - & 0,0 & - & 0,0 & - & 0,0 \\
\hline Pilar do Sul & - & 0,0 & - & 0,0 & - & 0,0 & - & 0,0 & - & 0,0 \\
\hline Sorocaba & 12 & 26,7 & 10 & 23,3 & 8 & 11,6 & 12 & 20,7 & 7 & 9,7 \\
\hline São Paulo (coord leste) & 39 & 53,4 & 21 & 42,9 & 30 & 37,0 & 14 & 26,9 & 18 & 31,6 \\
\hline
\end{tabular}

O município de Sorocaba se diferencia por apresentar a partir do ano de 2003 um aumento no número de casos detectados, sendo Que, o número total de casos diagnosticados como PB foi significativamente maior do Que os casos MB.

Verificou-se Que a maioria dos municípios apresentou uma redução na detecção ao longo dos cinco anos, com destaQue para os municípios de Pilar do Sul e Pariquera-Açu Que apresentaram, no ano de 2005, registro zero de casos novos. Essa situação, contudo, precisa ser analisada com algumas reservas, tendo em vista Que fatores operacionais como: a baixa cobertura de serviços, a existência de dificuldades de acesso dos pacientes aos serviços ou o desconhecimento da doença na população, podem provocar a prevalência oculta ${ }^{(7)}$. A Coordenação Estadual do Programa de Hanseníase promoveu no Estado de São Paulo, em 1996, nos municípios sem detecção de hanseníase, várias atividades contidas no "Projeto Sinal de Alerta", como a capacitação dos profissionais e a divulgação de informações sobre a doença ${ }^{(8)}$.

Os dados da Tabela 2 mostram Que a porcentagem de casos com deformidade física entre o total de casos detectados e avaliados entre 2001 e 2005 , foi acentuada na maioria dos municípios estudados. Por outro lado, alguns municípios apresentaram uma redução acentuada dos casos com incapacidade e em Bauru, a percentagem de casos novos com incapacidade física, não apresentou mudanças no período de 2001 a 2005, indicando que metade dos casos novos apresentou incapacidade física.

O diagnóstico precoce e tratamento adequado são as melhores estratégias para prevenir a evolução da doença, das incapacidades físicas e sociais, bem como realizar o controle da doença. $\mathrm{O}$ grau de incapacidade física detectado à época do diagnóstico é um importante indicador operacional de avaliação do $\mathrm{PCH}^{(1)}$.

Pode-se observar na Tabela 3, Que as percentagens de avaliação entre os casos novos, de 2001 a 2005, não foi uniforme e constante em todos os municípios. O município de São José do Rio Preto apresentou uma redução, sendo esse declínio mais acentuado no ano de 2003; dois municípios, Sorocaba e José Bonifácio, apresentaram acréscimo no percentual de casos avaliados; e em Bauru a cobertura não foi alcançada apenas no ano de 2003.

$\mathrm{Na}$ Coordenadoria Leste da cidade de São Paulo, exceto em 2002, a percentagem de avaliados não ultrapassou $90 \%$ dos casos diagnosticados.

Segundo a Coordenadora do Programa Nacional de Controle da Hanseníase, da Secretaria de Vigilância em Saúde do Ministério da Saúde ${ }^{(9)}$, é preciso fazer mais... é urgente a melhora do atendimento nos serviços de saúde, e recomenda aos profissionais de saúde Que: " para diagnosticar, é preciso examinar todo o corpo da pessoa, apalpar os membros (braços e pernas), fazer um bom teste de sensibilidade superficial da pele, pedir ao paciente Que feche os olhos para ver se 
Tabela 3. Proporção da avaliação do Grau de incapacidade física realizada entre os casos detectados, 2001 a 2005, segundo os municípios estudados. São Paulo/SP. 2007.

\begin{tabular}{|c|c|c|c|c|c|c|c|c|c|c|}
\hline \multirow{3}{*}{ Municípios } & \multicolumn{10}{|c|}{ Proporção de avaliados com incapacidade física } \\
\hline & \multicolumn{2}{|c|}{2001} & \multicolumn{2}{|c|}{2002} & \multicolumn{2}{|c|}{2003} & \multicolumn{2}{|c|}{2004} & \multicolumn{2}{|c|}{2005} \\
\hline & $\mathrm{n}$ & $\%$ & $\mathbf{N}$ & $\%$ & $\mathrm{n}$ & $\%$ & $\mathrm{n}$ & $\%$ & $\mathrm{n}$ & $\%$ \\
\hline Bauru & 9 & 100,0 & 11 & 100,0 & 22 & 95,5 & 11 & 100,0 & 15 & 100,0 \\
\hline Presidente Prudente & 55 & 100,0 & 53 & 100,0 & 47 & 97,9 & 40 & 97,5 & 32 & 100,0 \\
\hline Dracena & 35 & 91,4 & 32 & 75,0 & 19 & 100,0 & 28 & 100,0 & 21 & 95,2 \\
\hline São José do Rio Preto & 50 & 98,6 & 43 & 90,7 & 36 & 66,7 & 46 & 82,6 & 26 & 80,7 \\
\hline José Bonifácio & 12 & 66,7 & 19 & 78,9 & 20 & 85,0 & 10 & 100,0 & 7 & 100,0 \\
\hline Catanduva & 24 & 62,5 & 9 & 100,0 & 10 & 90,0 & 12 & 91,7 & 17 & 82,3 \\
\hline Parieuea-Açu & 6 & 83,3 & 1 & 100,0 & 4 & 100,0 & 7 & 85,7 & - & 0,0 \\
\hline Pilar do Sul & - & 0,0 & - & 0,0 & - & 0,0 & - & 0,0 & - & 0,0 \\
\hline Sorocaba & 47 & 95,7 & 45 & 95,6 & 72 & 95,8 & 61 & 95,1 & 73 & 98,6 \\
\hline São Paulo (coord leste) & 60 & 82,2 & 46 & 93,9 & 69 & 85,2 & 39 & 75,0 & 50 & 87,7 \\
\hline
\end{tabular}

Tabela 4. Ações referidas como realizadas pelos profissionais nos serviços de saúde estudados. São Paulo /SP, 2007.

\begin{tabular}{cc}
\hline Ações realizadas nos serviços de saúde & $\mathbf{n}$ \\
Atendimentos rotina / gerência & 59 \\
Ações relativas ao PCH & 38 \\
Ações relacionadas aos demais Programas & 36 \\
Ações relativas à Vigilância em saúde & 12 \\
Atividades de PesQuisa Científica & 2 \\
\hline Tipo de Ações em hanseníase & 36 \\
\hline Tratamento & 23 \\
Diagnóstico & 22 \\
Prevenção de Incapacidade & 15 \\
Vigilância Epidemiológica & 7 \\
Educação em Saúde & 2 \\
Capacitação de Recursos Humanos & 2 \\
Pesquisa & \\
\hline
\end{tabular}

ele perdeu a sensibilidade à dor e ao calor, avaliar se há perda da força muscular de mãos e pés". Nesse sentido, o PCH tem buscado sensibilizar os gestores municipais e estaduais para Que ampliem o atendimento. E conclui afirmando que "é preciso reconhecer que essa é uma doença endêmica de forte impacto na saúde do indivíduo"(9).

\section{Profissionais e Ações Desenvolvidas nos Serviços de Saúde}

Os dados apresentados a seguir apresentam uma descrição das características dos profissionais assistenciais entrevistados, e das ações referidas por eles como aQuelas desenvolvidas nos seus locais de trabalho.

Para a realização da análise das ações, tomou-se como referência a Resolução SS - 130 de 2001 Que aprova a Norma Técnica Que estabelece as Diretrizes e Estratégias para as ações de controle da hanseníase e preconiza esQuemas de tratamento ${ }^{(3)}$.

\section{Profissionais: algumas características}

No total foram entrevistados 59 profissionais Que trabalhavam nos serviços de saúde estudados, a maioria $(67,8 \%)$ dos profissionais tinha formação profissional universitária e os demais $(32,2 \%)$ tinha formação de nível médio e elementar.

Os dados evidenciam Que foram 3 categorias profissionais as mais pesquisadas: enfermeira $(33,9 \%)$, auxiliar de enfermagem (22\%) e médico (20,3\%), dos Quais, 8,5\% eram médicos dermatologistas. Da totalidade dos profissionais, 53 (90\%) exerciam ações de assistência em hanseníase, 3 (5, I \%) ocupavam cargos de gestão em vigilância epidemiológica, $2(3,4 \%)$ eram gerentes de serviços de saúde, e 2 profissionais ocupavam o cargo de pesquisador e exerciam também atividades assistenciais. Cabe destacar que os profissionais Que ocupavam os cargos de gerente dos serviços de saúde realizavam ações assistenciais em hanseníase esporadicamente Quando necessário.

Dos entrevistados, a metade (5 I\%) tinha um tempo superior a 15 anos de formado, nenhum era formado há menos de um ano e $19 \%$ tinha entre 5 a 10 anos de formação profissional. Ressalta-se, assim, relevante experiência profissional na equipe Que trabalha com a assistência à hanseníase.

Em relação ao tempo de exercício nos serviços de saúde, 53,4\% trabalhava há mais de 5 anos no local, dos Quais 20,3\% tinha um tempo superior a 15 anos de exercício de trabalho nos serviços estudados; 10, 1\% trabalhavam há um tempo inferior a I ano.

Dentre os estudados, 40,7\% referiu uma experiência profissional 
em hanseníase entre I e 5 anos, 22\% acima de 15 anos e 8,5\% trabalha com hanseníase há menos de um ano, mais Que a metade $(57,6 \%)$, tinha uma jornada de trabalho correspondente a 40 horas semanais.

\section{As Ações de Controle da Hanseníase}

$\mathrm{O}$ PCH normatiza as ações a serem desenvolvidas pelos profissionais de saúde visando a redução da morbidade da doença para menos de 1/10.000 habitantes; tem como objetivos detectar precocemente os casos novos, evitar a transmissão da doença aos menores de 15 anos de idade e a reduzir os danos causados pela doença, as incapacidades físicas e psicossociais.

O Ministério da Saúde preconizou cinco ações programáticas para controlar e eliminar a endemia da hanseníase em nosso país: ações de detecção de casos/diagnóstico; tratamento; vigilância de contatos; prevenção das incapacidades físicas e educação em saúde ${ }^{(2)}$.

Dessa forma, as Questões abordadas, a seguir, estão relacionadas às interfaces da implantação da política assistencial em hanseníase e a forma de trabalhar dos profissionais envolvidos na organização e produção assistencial dos serviços de saúde, entendendo-se assim, as ações assistenciais, gerenciais e de planejamento como resultado de um trabalho.

A maioria dos profissionais entrevistados assume responsabilidades além das inerentes ao PCH, dentre as Quais as do Programa da Criança, Mulher, Tuberculose e Aids.

$\mathrm{Na}$ Tabela 4 observa-se Que dentre as ações desenvolvidas relacionadas à hanseníase, as mais citadas pelos profissionais foram as relativas ao tratamento da doença, diagnóstico, seguida das ações de prevenção de incapacidade. Quanto ao desenvolvimento e implementação da Vigilância Epidemiológica foram mais citadas: preenchimento das fichas de notificação, coleta de dados, busca ativa de doentes e de seus contatos.

Em relação ao tratamento foram citadas as ações Que envolvem o trabalho da equipe, anamnese, avaliação funcional do doente, prescrição, orientações sobre doença e tratamento, entrega dos medicamentos e o controle do doente evitando que ele interrompa ou abandone o tratamento.

Quanto às ações de prevenção de incapacidade os profissionais dos serviços de saúde referiram Que as ações de avaliação e classificação do grau de incapacidade do doente, no momento da consulta pós diagnóstico, foram as mais citadas como realizadas nos serviços estudados. Entretanto, as ações Que envolvem o tratamento das incapacidades, bem como, as de educação em saúde foram pouco citadas como desenvolvidas pelos pesquisados.

De acordo com os profissionais, o processo de constituição e funcionamento das equipes de saúde não possibilita, eficientemente, maior abrangência no controle da hanseníase, em especial nas ações de prevenção, controle, tratamento e recuperação/reabilitação dos doentes e ex-doentes com hanseníase. Iustifica-se esse entendimento, por meio dos depoimentos dos pesquisados, Que evidenciaram Que nos serviços de saúde existem vários profissionais, de diferentes categorias, Que realizam ações isoladas, muitas vezes, sem articulação e sem comunicação.

Atualmente existe uma maior organização dos serviços, tanto na estrutura física, como de recursos humanos, entretanto não existe uma integração ou efetivação do trabalho em equipe, o Que acaba corroborando com uma assistência menos acolhedora, ou seja, ela se torna mais fragmentada

\section{As Unidades de Saúde Estudadas}

Quase a totalidade, 93,2\%, dos profissionais pesquisados estava inserida em serviços de saúde de gestão municipal ou municipalizada. Quanto ao tipo de serviços, a maioria $(52,5 \%)$ estava em unidades de atenção básica, (PSF 20,3\% e UBS tradicional 32,2\%), os demais em unidades de referência $(47,4 \%)$.

O trabalho desenvolvido pelos profissionais pesquisados está diretamente relacionado à estrutura e à forma de organização dos serviços de saúde. Os serviços de saúde de menor complexidade não estão organizados para realizar as ações contidas no $\mathrm{PCH}$, acolhem ou fazem a identificação dos casos suspeitos com hanseníase em sua rotina de trabalho na unidade e realizam o encaminhamento dos casos suspeitos para os serviços de saúde de maior complexidade, Quase sempre para as Unidades de Saúde de Referência do município.

A Resolução SS - de 2001 estabelece dentre as ações de organização dos serviços de controle da hanseníase que: "a rede de serviços do SUS - São Paulo deverá ser organizada de forma a Que todo caso suspeito de hanseníase tenha acesso a serviços para a assistência integral em todos os níveis de complexidade e, para tanto, as regionais de saúde deverão identificar os serviços para as ações de suspeição, diagnóstico e tratamento, bem como identificar e organizar pelo menos um serviço ambulatorial de referência em cada Diretoria Regional de Saúde”(3).

Uma característica observada nos depoimentos dos entrevistados é Que na maioria dos serviços de saúde Que tem o PSF, não está implantado o $\mathrm{PCH}$, porém os profissionais receberam orientações sobre hanseníase, na possibilidade de facilitar a identificação dos casos suspeitos e fazer o encaminhamento às unidades de saúde da sua região onde existe o atendimento à hanseníase.

Os serviços de saúde Que são considerados como referência para hanseníase recebem os casos encaminhados, Que podem ser do próprio município, de municípios vizinhos ou de outros municípios mais distantes. Em conformidade com os depoimentos dos profissionais, lotados nos serviços de referência, os encaminhamentos são realizados pelos profissionais médicos Que solicitam a elucidação do diagnóstico ou tratamento, e em especial dos casos com reação hansênica, ou seja, dos casos considerados complexos.

Em relação ao sistema de referência e contra-referência a grande maioria dos profissionais respondeu Que, está implantado entre os serviços de saúde, e para uma pequena parte dos profissionais, a referência existe formalmente a partir de um acordo entre os municípios. As referências mais citadas, para encaminhamento do doente foram os Ambulatórios de Especialidades e, em seguida, os serviços de saúde de outros municípios e o Instituto Lauro de Souza Lima (ILSL) para o atendimento das necessidades complexas e a realização de curativos; referem a ausência de normas e orientações nos serviços para a realização do encaminhamento dos doentes.

Quanto à contra-referência, Quase a totalidade dos profissionais referiu Que desconhece o significado desta etapa do processo; a contra-referência, para a maioria, não ocorre e Que Quando ocorre é por meio de comunicação verbal.

Nos casos de internação hospitalar, a maioria dos profissionais referiu Que, em primeiro lugar, fazem o encaminhamento para os hospitais gerais e em segundo lugar para os hospitais especializados. 
O ILSL foi predominantemente caracterizado como um grande responsável pelo o desenvolvimento profissional, com destaque para a assistência, tratamento e a reinserção social dos doentes com incapacidade física. Cabe destacar Que esse Instituto foi reconhecido pelo Ministério da Saúde como referência hospitalar especializada para todo o Estado de São Paulo além de se constituir em Centro de Referência Nacional em Hanseníase ${ }^{(3)}$.

A integração do serviço com o ensino, em especial com as universidades e faculdades de medicina, enfermagem e fisioterapia não está fortemente estabelecida. Nos serviços de maior complexidade, essa integração ocorre como campo de estágio, tanto em relação às práticas assistenciais como em relação às ações de vigilância epidemiológica. Em contrapartida, as faculdades oferecem aos trabalhadores cursos de capacitações ou encontros visando a melhoria na atenção à hanseníase.

Os profissionais das regiões Norte e Oeste referiram Que existe um trabalho assistencial em hanseníase realizado em conjunto com os profissionais do ILSL. Da mesma forma, os profissionais da região Sul e na Capital do Estado referiram desenvolver projetos assistenciais com a comunidade em parceria com a Fundação Paulista de Combate à Hanseníase localizada na cidade de São Paulo, com atividades Que visam a reinserção do doente no mercado de trabalho, por meio de cursos de: corte costura, cabeleireiro, artesanato e auxiliar de enfermagem. Em Sorocaba, por meio da verba municipal e auxilio de voluntários, no final do ano, é realizado o curso de confecção de cartões de natal, com a finalidade de propiciar uma assistência psicológica e peQuena remuneração aos doentes de hanseníase e de tuberculose.

No município de São José do Rio Preto, considerado de alta incidência em hanseníase, está sendo desenvolvido um projeto, visando a busca de todos os contatos dos pacientes Que fizeram tratamento naQuele serviço a partir 1998.

Segundo informações dos profissionais, o Hospital Regional do Vale do Ribeira localizado no município de Parieuera Açu, considerado como referência em hanseníase para 22 municípios da região, desenvolveu nos últimos anos, uma tecnologia mais avançada em serviços de atendimento de urgência, valorizando a assistência a traumas, deixando de desenvolver ações de outros agravos relevantes no município, como hanseníase.

Segundo a proposta da Secretaria de Estado da Saúde, o centro de referência deveria ter uma estrutura administrativa Que propiciasse uma maior autonomia, agilidade e resolutividade no atendimento às necessidades para o desenvolvimento do atendimento. Para tanto, propõe Que a estrutura física seja contemplada com instalações adeeuadas para o desempenho de suas atividades.

Neste aspecto, apenas os centros de referência dos municípios estudados estão próximos a essa proposta. Em conformidade com o conteúdo obtido nas entrevistas as áreas físicas de todas as unidades não possibilitam o desenvolvimento integral das atividades assistenciais com Qualidade. O número de salas é insuficiente frente ao número de profissionais e da demanda existente nos serviços, situação Que se agrava em alguns serviços em Que em uma única sala funciona a administração, a farmácia e ainda a pós-consulta; em outra sala ao lado funciona a emergência, os curativos, a esterilização e o expurgo, simultaneamente. Em outros serviços os profissionais referiram a necessidade de colocar nas portas das salas um aviso de Que as salas estão ocupadas para a realização de determinadas atividades, para dessa forma poder realizar a prática sem haver interrupção.

De acordo com os depoimentos, podemos verificar Que as salas são inadequadas insuficientes e na maioria dos serviços não há salas para a técnica de curativo, bem como sala para ações educativas com os doentes. Por outro lado, a minoria dos profissionais estudados, gerentes e coordenadores da hanseníase, referiu Que a estrutura física das UBS é adequada e Que as salas para a assistência são suficientes, destacando a necessidade de melhorar a estrutura geral das salas.

Na maioria dos serviços de saúde de média complexidade, a área física existente para o desenvolvimento das atividades em hanseníase, consiste de três salas: uma onde são realizadas as consultas médicas, outra para todos os procedimentos de acolhimento, orientações, pré e pós consulta e uma terceira onde são feitos os curativos dos doentes com hanseníase.

Quanto aos recursos Que os serviços de saúde ou PCH dispõem para apoiar os pacientes frente às suas necessidades evidencia-se Que o auxílio para o transporte dos doentes foi o mais citado pelos profissionais pesquisados, no entanto o vale-transporte em muitos serviços de saúde é fornecido para os doentes de uma forma geral não se constituindo em um benefício específico para os doentes de hanseníase. Em alguns serviços são utilizadas ambulâncias para o transporte dos doentes para locais distantes do município. Também foi citado o fornecimento da cesta básica e de calçados adaptados, estes direcionados especificamente aos doentes Que apresentam seeüelas ou perda de sensibilidade nos pés.

Segundo os profissionais entrevistados em relação a material para curativos, em alguns serviços de saúde do interior do Estado, existe falta ou escassez do mesmo. Quanto aos insumos Que possibilitam a realização do diagnóstico, tratamento e ações de prevenção de incapacidade física foram referidos como suficientes. O material de educação da hanseníase à comunidade é recebido nos dias Que antecedem a campanha.

A capacitação de pessoal vem sendo priorizada e intensificada para os profissionais do PSF, visando o preparo para identificar manchas suspeitas e realizar encaminhamento para o serviço municipal de saúde de referência. A capacitação dos profissionais Que realizam assistência em hanseníase constitui um dos resultados esperados, a fim de sustentar a eliminação da hanseníase enQuanto problema de saúde pública nos municípios ${ }^{(1)}$.

Os relatos dos profissionais, do município Que atingiu a meta de eliminação da hanseníase, apontam Que não existe organização do serviço de saúde e capacitação dos profissionais, para atender as ações preconizadas para os municípios sem detecção, e em especial não é realizada a busca ativa de casos de hanseníase, o Que causa preocupação e Questionamento sobre a real situação de casos ativos de hanseníase naquela localidade.

Em relação às mudanças Que ocorreram no atendimento aos doentes nos últimos cinco anos, após a Resolução SS 130 de 8/10/ 2001, para os serviços de menor complexidade não ocorreram mudanças significativas Quanto à assistência em hanseníase. Já para os serviços de especialidades foram atribuídas transformações Que permitiram maior acesso ao tratamento psicológico, bem como o aumento do número de profissionais capacitados, o que possibilitou uma assistência de melhor Qualidade. Quanto à capacitação em hanseníase, Quase a metade dos profissionais dos serviços 
especializados, referiu possuir capacitação na área, por meio de especializações e de cursos oferecidos pelas regionais de saúde.

Para Que se alcance a meta de eliminação da hanseníase em nosso país, segundo o Ministério da Saúde ${ }^{(2)}$, "os serviços de saúde necessitam de profissionais capazes de trabalhar em equipe, interagir com as pessoas e grupos, adouirir e processar informações, comunicar-se e expressar suas idéias, além de serem capazes de utilizar conhecimentos e habilidades específicas para desempenhar as funções próprias do seu trabalho".

Em todos os serviços de saúde, foi possível verificar Que o sistema de informações é deficitário. $\mathrm{O}$ Que acarretou grandes dificuldades na obtenção de dados epidemiológicos da doença, além do fornecimento de dados defasados e não coincidentes com os dados oficiais contidos nas planilhas da Secretaria de Estado da Saúde. Esse fato contribui para um desconhecimento da real situação epidemiológica da hanseníase em seus locais de trabalho e na região. Couttolenc $^{(11)}$, já na década de 1980, reforçava a importância do sistema de informações afirmando ser elemento essencial no gerenciamento de um programa.

\section{Interlocutores: Algumas Características}

Os resultados apresentados a seguir foram obtidos por meio de entrevista, com os profissionais Que se denominaram responsáveis pela interlocução do nível estadual com a atenção básica nos municípios selecionados para o presente estudo, totalizando 17 interlocutores.

O profissional da saúde em hanseníase denominado de interlocutor é o articulador, facilitador entre os grupos de vigilância epidemiológica dos três níveis de atenção (federal, estadual/regional e municipal). Visa o controle da endemia por meio da avaliação dos dados epidemiológicos e operacionais, a elaboração de propostas de organização de serviços/referência e contra referência e a capacitação dos recursos humanos.

Quase a totalidade dos entrevistados $(16=94,1 \%)$ possuía formação universitária, assim distribuídos, 10 enfermeiras, 4 médicos, dos euais, 2 tinham a especialidade em pediatria, I educadora de saúde pública, I biólogo, I interlocutor era oficial administrativo com formação em nível médio. Mais Que a metade $(13=72,7 \%)$ tinha um tempo de formado superior a 15 anos e os demais estavam entre o período 5 a 15 anos.

A maioria dos interlocutores estava lotada na secretaria municipal de saúde e ocupavam cargos diretamente relacionados à direção, coordenação e supervisão da Vigilância Epidemiológica, outros eram responsáveis pela coordenação e planejamento dos programas de controle da hanseníase e da tuberculose e possuíam cargos de assistência técnica da equipe de coordenação da hanseníase e 3 eram gerentes de unidade básica de saúde.

Quase a metade $(8=47 \%)$ desempenhava o papel de interlocutor há um tempo inferior a 5 anos, $5(29,4 \%)$ trabalhavam entre 5 e 10 anos e $3(17,6 \%)$ há um período superior a 15 anos, sendo Que na área específica de hanseníase, 13 (16,5\%) atuavam há cerca de 10 anos, e $3(17,6 \%)$ tinham experiência profissional com hanseníase há mais de 15 anos, dos Quais, 47\% tinham uma jornada de trabalho de 30 horas semanais.

Dentre as atividades Que realizavam nos seus locais de trabalho, as mais citadas foram aQuelas Que estavam diretamente relacionadas à responsabilidade pelos dados do sistema de informações da regional municipal de saúde onde estavam lotados, bem como, as atividades de coordenação de programas e projetos em hanseníase e outros agravos, a capacitação de recursos humanos dos serviços de saúde, o controle epidemiológico das doenças, a coordenação administrativa e as ações de educação em saúde.

Dentre as atividades Que desenvolviam e Que estavam diretamente relacionadas à hanseníase, as mais citadas pelos interlocutores foram as ações de educação em saúde e as relacionadas à vigilância epidemiológica em hanseníase, em seguida, foram referidas as atividades relacionadas ao diagnóstico e ao tratamento da hanseníase, prevenção de incapacidades e encaminhamentos dos doentes. A atividade de pesquisa foi referida por apenas um interlocutor. Quanto às ações de educação em saúde, segundo relato de alguns interlocutores a mesma não é realizada de forma sistematizada, sendo desenvolvida apenas nas campanhas anuais.

Pode-se verificar a preocupação Que existe, por parte dos interlocutores com os dados oriundos dos serviços de saúde em relação ao controle de comunicantes e à busca ativa dos doentes. Por outro lado, evidencia-se uma fragilidade no preenchimento correto das informações referentes às ações desenvolvidas pelos profissionais.

Para os interlocutores, nos últimos anos, em alguns serviços de saúde houve uma reorganização de forma Que o atendimento específico para a prevenção de incapacidade passou a ser realizado em unidade específica como centro de reabilitação. Para um interlocutor esta mudança acarretou uma sobrecarga aos profissionais uma vez Que devem atender a todos os usuários do SUS e os centros estão localizados distantes dos locais de atendimento do $\mathrm{PCH}$, o Que foi referido como fatores dificultadores do acompanhamento do atendimento dos casos. Alguns relataram a dificuldade da efetivação da contra referência, o Que dificulta o acompanhamento dos casos.

Quanto à assessoria da Regional de Saúde prestada aos serviços de saúde elas são realizadas por meio de normas e instruções assim como na avaliação e no controle da doença e dos serviços oferecidos/ prestados à população e no desenvolvimento de capacitação de recursos humanos.

A insuficiência registrada Quanto aos recursos humanos foi mencionada por $37,5 \%$ dos interlocutores, apontando ainda Que muitos dos profissionais Que trabalham na unidade têm muito tempo de serviço e estão prestes a se aposentarem, sem plano de capacitação visando preparar novos profissionais para atuarem na área.

Em relação aos recursos de apoio e auxílio Que os doentes com hanseníase recebem durante o tratamento da doença para a concretização dos cuidados à saúde, o fornecimento de passes para o transporte à unidade de saúde foi referido por todos os interlocutores, as cestas básicas são fornecidas por alguns serviços de saúde, bem como a confecção de palmilhas e calçados nos casos indicados, sendo Que para esse último recorre-se à unidade especializada do HLSL por meio de encaminhamento.

Alguns interlocutores compreendem Que a grande maioria dos serviços de saúde possui uma área física para o atendimento da hanseníase em condições precárias e com pouco espaço físico para o desenvolvimento de todas as atividades previstas no $\mathrm{PCH}$. Referiram Que a assistência à hanseníase Quase sempre é realizada em prédios de uma unidade de saúde com atendimento especializado em saúde, com entrada separada para o atendimento da hanseníase/tuberculose, 
e geralmente comportam salas para o atendimento de enfermagem, realização de curativos, consultório médico, arquivo dos prontuários dos pacientes e documentação, bem como salas para as ações de vigilância epidemiológica. As ações de prevenção e tratamento das incapacidades, assim como as avaliações rotineiras são realizadas na própria unidade ou em outra unidade de saúde, Quase sempre distantes do serviço de saúde Que iniciou o tratamento.

Essa característica da área física corresponde ao antigo modelo das unidades de saúde onde a área de atendimento da hanseníase sempre se localizava em local mais externo, Quando não com acesso independente, o que nas unidades mais antigas, se mantém até hoje. Isso é reforçado pela ausência de alteração no modelo gerencial em Que todos os prontuários dessa área são arQuivados no próprio setor.

\section{CONSIDERAÇÕES FINAIS}

Os resultados obtidos no presente estudo permitiram evidenciar Que nos serviços de saúde estudados o desenvolvimento de ações destinadas à redução da morbi-mortalidade por hanseníase, no âmbito do Sistema Único de Saúde (SUS) - São Paulo, não apresentou mudanças significativas na dinâmica da reorganização desses serviços de saúde, após a publicação da Resolução SS 130/2001.

As ações de controle da hanseníase nos serviços de saúde de maior complexidade são realizadas por profissionais com experiência significativa de trabalho. $\mathrm{Na}$ Quase maioria dos serviços, existe um desconhecimento por parte dos membros da equipe, do trabalho desenvolvido pelos profissionais do próprio grupo.

A equipe multiprofissional responsável pela assistência dos doentes com hanseníase, nos serviços estudados, varia de acordo com o perfil da unidade de saúde devido a sua complexidade. A evidente necessidade de capacitação desses profissionais torna-se instrumento fundamental para realizar as ações preconizadas pelo Programa de Controle da Hanseníase. No estado de São Paulo tal capacitação está fortemente relacionada com a presença do Instituto Lauro de Souza Lima, uma vez Que este é um grande pólo de produção do conhecimento e pesquisa, facilitando o acesso a cursos, atualizações e treinamentos nessa área.

Apesar da reconhecida importância da execução constante de ações de educação em saúde como prática Que propicia a adesão ao tratamento e a emancipação do sujeito com hanseníase, devido à superação de limitações provocadas pela doença, observou-se Que as mesmas não são sistemáticas e nem citadas com algum grau de prioridade pelos sujeitos pesquisados.

Soma-se a isso, a necessidade de implementação da busca ativa no controle da hanseníase para a detecção de casos novos e a possibilidade do diagnóstico precoce, visto Que, os dados epidemiológicos, indicam a realização tardia do diagnóstico de hanseníase na grande maioria dos serviços de saúde estudados, isto contribui para um risco significativo dos doentes apresentarem incapacidades físicas e/ou deformidades, reforçando a necessidade de organização de um fluxo de referência das ações de prevenção de incapacidades, em todos os níveis de atenção.

Um outro fato Que deve ser mencionado está relacionado ao sistema de informações dos dados epidemiológicos, Que dada sua importância, apresenta em todos os serviços e regionais de saúde déficits e lacunas que deveriam ser melhorados.

Espera-se Que os resultados deste estudo sirvam de fontes de conhecimento, visando a melhoria das ações de controle à hanseníase, minimizando o Quadro do doente e da doença e impulsionem novas investigações.

\section{REFERÊNCIAS}

I. Ministério da Saúde (BR). Secretaria de Vigilância em Saúde. Departamento de Vigilância Epidemiológica. Programa Nacional de Eliminação da Hanseníase. Plano Nacional de Eliminação da Hanseníase em nível municipal 2006-20 I0. Brasília: Ministério da Saúde; 2006

2. Ministério da Saúde (BR). Secretaria de Políticas de Saúde. Área de Dermatologia Sanitária. Diretrizes Nacionais para a Elaboração de Programas de Capacitação para a Equipe de saúde da rede básica atuar nas ações de controle da hanseníase. Brasília: MInistério da Saúde; 2000

3. Estado de São Paulo. Secretaria de Estado da Saúde de São Paulo. Resolução SS-130, de 08/10/200 I. Aprova a Norma Técnica Que estabelece as diretrizes e estratégias para as ações de controle da hanseníase e preconiza esquemas de tratamento. Diário Oficial do Estado de São Paulo 200 l out 10; 19- 22.

4. Estado de São Paulo. Secretaria de Estado da Saúde de São Paulo. Centro de Vigilância Epidemiológica Prof. Alexandre Vranjac - CVE. Divisão de Vigilância Epidemiológica da Hanseníase. Plano de Ação para o combate à Hanseníase no Estado de São Paulo para o período de 2006 a 20I0. São Paulo: Imprensa Oficial; 2006.

5. Carvalho GI, Santos L. Sistema Único de Saúde. Comentários à Lei Orgânica da Saúde (Leis 8.080/90 e 8.142/90). São Paulo: Hucitec; 1995.

6. Carvalho SR. Saúde coletiva e promoção da saúde: sujeito e mudança. São Paulo: Hucitec; 2005

7. Estado de São Paulo. Secretaria de Estado da Saúde de São Paulo. Centro de Vigilância Epidemiológica Prof. Alexandre Vranjac - CVE. Divisão de Vigilância Epidemiológica da Hanseníase. Plano de Ação para o combate à Hanseníase no Estado de São Paulo para o período de 2003 a 2006. São Paulo: Imprensa Oficial; 2002.

8. World Health Organization. Global leprosy situation, 2006. Disponível em: http://www.who.int/lep/Reports/GlobalStrategyPDF-verison.pdf

9. Nogueira W, Gonçalves OSI, Lessa Z. Implantação de procedimentos especiais de vigilância epidemiológica em municípios sem detecção de casos. Int I Leprosy 1988; 66.

10. Oliveira MLW Hanseníase RADIS. Comun Saúde 2008; (68): 10-3.

II Couttolenc BF. O papel da análise custo-efetividade no processo decisório em saúde: o caso do sub programa de controle da hanseníase [dissertação]. São Paulo (SP): Escola de Administração de Empresas de São Paulo; 1987. 